\title{
Mid-infrared sizes of circumstellar disks around Herbig Ae/Be stars measured with MIDI on the VLTI*
}

Ch. Leinert ${ }^{1}$, R. van Boekel ${ }^{2}$, L. B. F. M. Waters ${ }^{2,3}$, O. Chesneau ${ }^{1}$, F. Malbet ${ }^{4}$, R. Köhler ${ }^{1}$, W. Jaffe ${ }^{5}$, Th. Ratzka ${ }^{1}$, A. Dutrey ${ }^{4}$, Th. Preibisch ${ }^{6}$, U. Graser ${ }^{1}$, E. Bakker ${ }^{5}$, G. Chagnon ${ }^{8}$, W. D. Cotton ${ }^{9}$, C. Dominik ${ }^{2}$, C. P. Dullemond ${ }^{7}$, A. W. Glazenborg-Kluttig ${ }^{10}$, A. Glindemann ${ }^{11}$, Th. Henning ${ }^{1}$, K.-H. Hofmann ${ }^{6}$, J. de Jong ${ }^{5}$, R. Lenzen ${ }^{1}$, S. Ligori ${ }^{1}$, B. Lopez ${ }^{12}$, J. Meisner ${ }^{5}$, S. Morel ${ }^{11}$, F. Paresce ${ }^{11}$, J.-W. Pel ${ }^{13}$, I. Percheron ${ }^{11}$, G. Perrin ${ }^{8}$, F. Przygodda ${ }^{1}$, A. Richichi ${ }^{11}$, M. Schöller ${ }^{11}$, P. Schuller ${ }^{14}$, B. Stecklum ${ }^{15}$, M. E. van den Ancker ${ }^{2}$, O. von der Lühe ${ }^{16}$, and G. Weigelt ${ }^{6}$

\footnotetext{
${ }^{1}$ Max-Planck-Institut für Astronomie, Königstuhl 17, 69117 Heidelberg, Germany e-mail: leinert@mpia-hd.mpg.de

2 Sterrenkundig Instituut "Anton Pannekoek", Kruislaan 403, 1098 SJ Amsterdam, The Netherlands

3 Instituut voor Sterrenkunde, Celestijnenlaan 200B, 3001 Leuven, Belgium

${ }^{4}$ Laboratoire d'Astrophysique, Observatoire de Grenoble, BP 53, 38041 Grenoble Cedex 9, France

5 Sterrewacht Leiden, Niels-Bohr-Weg 2, 2300 RA Leiden, The Netherlands

6 Max-Planck-Institut für Radioastronomie, Auf dem Hügel 69, 53121 Bonn, Germany

7 Max-Planck-Institut für Astrophysik, Karl-Scharzschild-Straße 1, 85741 Garching, Germany

8 Laboratoire d'Études Spatiales et d'Instrumentation en Astrophysique, Observatoire de Meudon, 5 place Jules Janssen, 92190 Meudon, France

9 National Radio Astronomy Observatory, 520 Edgemont Road, Charlottesville, VA 22903-2475, USA

10 ASTRON, Oude Hoogeveesnsedijk 4, PO Box 2, 7990 AA Dwingeloo, The Netherlands

11 European Southern Observatory, Karl-Schwarzschild-Strasse 2, 85748 Garching, Germany

12 Observatoire de la Côte d'Azur, Boulevard de l'Observatoire, BP 4229, 06304 Nice Cedex 4, France

13 Kapteyn Institute, Landleven 12, Postbus 800, 9700 AV Groningen, The Netherlands

14 Harvard-Smithonian Center for Astrophysics, 60 Garden Street, Cambridge, MA 02138, USA

15 Thüringer Landessternwarte Tautenburg, Sternwarte 5, 07778 Tautenburg, Germany

16 Kiepenheuer-Institut für Sonnenforschung, Schöneckstr.6, 79104 Freiburg, Germany
}

Received 1 February 2004 / Accepted 9 April 2004

\begin{abstract}
We present the first long baseline mid-infrared interferometric observations of the circumstellar disks surrounding Herbig Ae/Be stars. The observations were obtained using the mid-infrared interferometric instrument MIDI at the European Southern Observatory (ESO) Very Large Telescope Interferometer VLTI on Cerro Paranal. The $102 \mathrm{~m}$ baseline given by the telescopes UT1 and UT3 was employed, which provides a maximum full spatial resolution of 20 milli-arcsec (mas) at a wavelength of $10 \mu \mathrm{m}$. The interferometric signal was spectrally dispersed at a resolution of 30, giving spectrally resolved visibility information from $8 \mu \mathrm{m}$ to $13.5 \mu \mathrm{m}$. We observed seven nearby Herbig Ae/Be stars and resolved all objects. The warm dust disk of HD 100546 could even be resolved in single-telescope imaging. Characteristic dimensions of the emitting regions at $10 \mu \mathrm{m}$ are found to be from $1 \mathrm{AU}$ to $10 \mathrm{AU}$. The $10 \mu \mathrm{m}$ sizes of our sample stars correlate with the slope of the $10-25 \mu \mathrm{m}$ infrared spectrum in the sense that the reddest objects are the largest ones. Such a correlation would be consistent with a different geometry in terms of flaring or flat (self-shadowed) disks for sources with strong or moderate mid-infrared excess, respectively. We compare the observed spectrally resolved visibilities with predictions based on existing models of passive centrally irradiated hydrostatic disks made to fit the SEDs of the observed stars. We find broad qualitative agreement of the spectral shape of visibilities corresponding to these models with our observations. Quantitatively, there are discrepancies that show the need for a next step in modelling of circumstellar disks, satisfying both the spatial constraints such as are now available from the MIDI observations and the flux constraints from the SEDs in a consistent way.
\end{abstract}

Key words. stars: circumstellar matter - techniques: interferometric - stars: formation - stars: pre-main-sequence infrared: stars

^ Based on observations made with the Very Large Telescope Interferometer at Paranal Observatory.

\section{Introduction}

Most young low- and intermediate mass stars are surrounded by a disk of gas and dust, which is believed to be an evolved 
form of the accretion disk from which the star formed. This disk dissipates on a typical timescale of $10^{7}$ years and is believed to be the site of planet formation. The structure and evolution of such supposedly proto-planetary disks is one of the focal points of current star- and planet formation studies. Since the material in these disks is cool, and their size is of the order of $100 \mathrm{AU}$ (corresponding to an angular size of 1 arcsec at the distance of the nearest low-mass star forming regions), the study of star- and planet formation requires high angular resolution observations at infrared and millimeter wavelengths.

After a decade-long concentration of efforts on the study of disks around the low-mass T Tauri stars, in recent years substantial progress has been made in high angular resolution studies of the disks surrounding Herbig Ae/Be stars, too. These are intermediate mass pre-main sequence stars, first defined as a group by Herbig (1960). By their ultraviolet radiation, some Herbig Ae/Be stars excite noticeable emission from the infrared bands of polycyclic aromatic hydrocarbon molecules (PAHs, see Malfait et al. 1998a), emissions which otherwise are ubiquitous in the diffuse interstellar medium (Mattila et al. 1996) and compact HII regions (Roelfsema et al. 1996). But the infrared spectrum of Herbig Ae/Be stars is mainly characterized by a large excess from dust in their circumstellar environment (Hillenbrand et al. 1992; Malfait et al. 1998b). In general infrared and mm emission from Herbig Ae/Be stars of earlier spectral type lacks clear indications of circumstellar disks while these are present in the $\mathrm{mm}$ interferometric observations of the later spectral types of this group (Natta et al. 2000). For the subset of Herbig Ae/Be stars with spectral type A or late B ("Herbig Ae stars") defined by the study of Malfait et al. (1998b), which is "isolated", i.e. not closely associated with molecular cloud material, the infrared and $\mathrm{mm}$ emission can safely be attributed to a circumstellar disk.

This is particularly evident at millimeter wavelengths, where spatially resolved images of isolated Herbig Ae stars convincingly show a disk signature at typical scales of several 100 AU in CO (Mannings \& Sargent 1997; Dutrey 2004). In the visible and near-IR, scattered light images reveal the presence of non-spherical dust distributions with a scale of up to $1000 \mathrm{AU}$ (e.g. Grady et al. 2001). In parallel, the latetype Herbig Ae/Be stars tend to show substantially smaller near-IR scattering haloes than their more massive early-type Herbig Ae/Be counterparts, probably reflecting differences in UV radiation field and evolutionary time scales (Leinert et al. 2001). Recent interferometric observations with the Palomar Testbed Interferometer at $2.2 \mu \mathrm{m}$ indicate that also the hottest dust in Herbig Ae stars has a non-spherical spatial distribution (Eisner et al. 2003), again suggesting a disk geometry. Most authors agree that the millimeter emission observed in Herbig Ae stars stems from cold grains in the mid-plane of an optically thick, gas-rich, usually passive, externally heated disk, while the mid-IR emission arises from the warm optically thin disk atmosphere (D'Alessio et al. 2001; Chiang \& Goldreich 1997).

Little is known however about the geometrical shape of the disks. The usual approach is indirect through attempts to fit the observed spectral energy distribution (SED). Hillenbrand (1992) had used, in analogy to the classification of T Tauri stars, the slope of infrared emission beyond $2.2 \mu \mathrm{m}$ to distinguish three groups of Herbig $\mathrm{Ae} / \mathrm{Be}$ stars. More relevant to our observations is the approach by Meeus et al. (2001) who concentrate on Herbig Ae stars and classified them into two groups, based on the slope of the 10-60 $\mu \mathrm{m}$ spectral region. Their group I sources show a rising IR spectrum and are interpreted in terms of flaring disks. The group II sources have a bluer spectral slope, which Meeus et al. (2001) propose is due to non-flaring disks. This interpretation is supported by the stronger emission in group I sources from PAHs: flaring disks subtend a larger solid angle and thus stellar photons can reach a larger surface area, resulting in stronger PAH emission.

A physical description of the qualitative picture drawn by Meeus et al. (2001) has been given recently in a series of papers by Dullemond et al. (2001) (hereafter DDN), Dullemond (2002) and Dominik et al. (2003). The DDN models are a modification of the Chiang \& Goldreich (1997, hereafter CG97) passive, centrally irradiated hydrostatic equilibrium flaring disk models for $\mathrm{T}$ Tauri stars. The most important feature of the DDN models is a puffed-up inner rim at the dust sublimation radius, which casts a shadow on the surface of the disk, substantially reducing the disk surface temperature behind the inner rim. This shadow affects the spatial scale of the mid-IR emission in a different way for flaring and non-flaring geometries and may be the basic principle giving rise to the two groups of Herbig Ae stars with different mid-infrared behaviour. While the DDN models are quite successful in explaining what they were made for, i.e. the infrared SEDs of HAe stars (Dominik et al. 2003), the underlying spatial distributions for these and other disk models finally will have to be based on high-spatial resolution observations of the circumstellar structures.

Recently, the Very Large Telescope Interferometer (VLTI, Glindemann et al. 2003) of ESO's Paranal Observatory has been equipped with MIDI, the MID-infrared Interferometric instrument (Leinert et al. 2003). MIDI combines the light of two telescopes and provides spectrally resolved visibilities in the $10 \mu \mathrm{m}$ atmospheric window. With the baselines between the $8.2 \mathrm{~m}$ - telescopes (UTs) of the order of $100 \mathrm{~m}$, the instrument will be most sensitive to source geometries a few AU in size for the distances of 100-300 pc typical of our sample. This is also the region from which most of the $10 \mu \mathrm{m}$ emission in the studied sources is thought to originate.

The instrument was successfully installed in late 2002, and first scientific observations were obtained in June of 2003. These observations were conducted in the context of both the MIDI consortium guaranteed time, and of the Science Demonstration Time, which was provided by ESO to demonstrate the science potential of the VLTI. Here we report on first results of MIDI observations of the disks surrounding Herbig Ae/Be stars. The paper is organized as follows: Sect. 2 describes the observations and data reduction procedures. In Sects. 3 and 4 we introduce the sample and present the results obtained on these sources. In Sect. 5 we discuss the spectrally resolved visibilities of the seven observed Herbig Ae/Be stars, correlate these with other known properties of the target stars and compare the observed visibilities with those predicted on the basis of DDN models. Section 6 summarizes the results of this study. 


\section{Observations and data reduction}

\subsection{Observing procedure}

The targets were observed in three nights of Guaranteed Time Observations and three nights of Science Demonstration Time from June 11-17, 2003. The individual observations are listed in Table 2. The observing sequence, typical of interferometric measurements, is influenced by the design of the instrument, presented in Leinert et al. (2003). After the coarse acquisition by the telescopes, we took images with MIDI in imaging mode, i.e. without beam combiner and without prism, resulting in one image per telescope beam. These separated single-telescope images were taken in parallel with the short wave $N$ band filter $(8.7 \mu \mathrm{m}$, width $1.4 \mu \mathrm{m})$ while chopping the secondary of the two involved UTs with typically $2 \mathrm{~Hz}$ over $10^{\prime \prime}$. The images were used to adjust the position of the stars to a predetermined pixel in order to maximize the overlap of both images for the following interferometric measurement. Then, the beam combiner, which produces two interferometric outputs of opposite signal, and the low resolution prism were introduced into the optical train. This prism gives a spectral resolution of $\lambda / \Delta \lambda \approx$ 30. To find the location of zero optical path difference (OPD), a range of a few millimeters around the expected point of path length equalisation was scanned. The scanning involved varying the setting of the VLTI delay lines while internally stepping the OPD rapidly over a few wavelengths. For unresolved or partially resolved sources, the "white light" fringe signal obtained by integrating the reading of each exposure over the complete wavelength range from $8 \mu \mathrm{m}$ to $13 \mu \mathrm{m}$ - clearly indicates the point of zero OPD. Then, an interferometric measurement with self-fringe tracking is started. In this mode, the piezo-mounted mirrors within MIDI are used to scan a range in OPD of six to eight wavelengths $(\lambda \approx 10 \mu \mathrm{m})$ in steps of typically $2 \mu \mathrm{m}$. After each scan, the position of the fringe packet in the scan is measured and the VLTI delay lines are adjusted in order to re-center the fringe packet for the next scan. In this way, typically a few hundred scans are obtained within 3$4 \mathrm{~min}$. Then, photometric data are recorded by blocking first the light from one, then from the other telescope and recording a few thousand frames in about a minute. For these photometric measurements, chopping of the telescopes is used again to obtain sky and background subtraction. No chopping is used during fringe search and tracking. The sensitiviy limit for this self-fringe tracking mode was $\approx 2 \mathrm{Jy}$ (unresolved source).

As usual, calibrator stars with known diameter were observed immediately after the object and in the same region of the sky to correct for the reduction in fringe contrast due to optical imperfections and atmospheric turbulence. The calibrators were taken from a list of 478 stars at least $5 \mathrm{Jy}$ bright at $10 \mu \mathrm{m}$ and selected for absence of circumstellar emission, disturbing companions or strong variability (see Table 3). The time lapse between object and calibrator observations was $30 \mathrm{~min}$. With the present accuracy of $\approx 10 \%$ per single visibility measurement, there is no problem using also calibrators observed in the same mode one or two hours earlier or later in connection with other objects.
It is an advantage of the long observing wavelength that these interferometric observations can be performed without a higher order adaptive optics correction even on the $8 \mathrm{~m}$ UT telescopes. For median seeing of $0.7^{\prime \prime}$ the Fried parameter has a value of $r_{0} \approx 5.3 \mathrm{~m}$. With the image stabilisation given by the tip-tilt sensors in the Coudé foci of the UTs we then have essentially diffraction-limited performance in the $10 \mu \mathrm{m}$ range. No adverse effect of the tip/tilt operation is seen in the interferometric data. But we disabled the active optics on the UTs during the interferometric measurement to avoid the OPD jumps of a few wavelengths associated with refocussing. The future higher order correction system MACAO will be helpful for data taken under less favourable seeing conditions.

\subsection{Data reduction}

For data reduction, a custom software written in the IDL language was used, based on power spectrum analysis. The first step is to read in the photometric datasets, average the frames on the target and the frames on the sky and subtract the average sky frame from the average target frame. This sky-subtracted frame contains the spectrum of the object, oriented horizontally on the chip. The position of the spectrum is measured columnwise by searching for peaks that are sufficiently high above the background fluctuations. The result is the position and width of the spectrum as a function of wavelength. This procedure is carried out independently on both photometric datasets (which contained data from telescopes UT1 and UT3, respectively). Then a mask is created with the average position and width of the two spectra as a function of wavelength.

This mask is used to extract the object data from the fringe tracking datasets. Each frame of the fringe data, corresponding to one individual OPD setting inside a scan, is reduced to a one-dimensional spectrum by multiplying by the mask and performing the weighted integral over the direction perpendicular to the spectral dispersion. Then the two - oppositely phased - interferometric output channels of the beam combiner are subtracted from each other. This combines the interferometric modulation of both channels into one and at the same time helps subtract the background. The few dozen spectra from each scan with the piezo-mounted mirrors are collected into a two-dimensional array with optical wavelength and OPD as axes. The contents of this array are column-wise Fouriertransformed from OPD to fringe frequency space. As a rule, four of the $\approx 0.05 \mu \mathrm{m}$ wide wavelength (pixel) channels were added to improve the signal-to-noise $(S / N)$ ratio. The fringe amplitude for each optical wavelength is then obtained from the power spectrum at the corresponding fringe frequency.

The scans where fringes were actually detected are selected based on the white-light fringe amplitude, i.e. the amplitude of fringes we see after integrating the signal over all usable wavelengths. The histogram of all white-light fringe amplitudes within a fringe track dataset usually shows a small peak near zero, and a broad peak at higher amplitudes. We interactively set a threshold just below this broad peak - for the faintest sources allowing self-fringe tracking this will correspond to $S / N \approx 2$ - and average the spectrally resolved fringe 
Table 1. Basic properties of the target stars.

\begin{tabular}{llccccccc}
\hline \hline Name & Sp. T. & $\begin{array}{c}T_{\text {eff }} \\
(\mathrm{K})\end{array}$ & $\begin{array}{c}\text { Luminosity } \\
\left(L_{\odot}\right)\end{array}$ & $\begin{array}{c}\text { Distance } \\
(\mathrm{pc})\end{array}$ & $\begin{array}{c}E_{B-V} \\
(\mathrm{mag})\end{array}$ & $\begin{array}{c}\log (\text { age }) \\
(\mathrm{yr})\end{array}$ & Group $^{1}$ & References \\
\hline HD 100546 & B9Vne & 10500 & 36 & $103 \pm 7$ & 0.09 & $>7.0$ & I & $2,5,6$ \\
HD 142527 & F7IIIe & 6260 & 31 & $200 \pm 50$ & 0.21 & $5.0 \pm 0.5$ & I $\rightarrow$ II & $2,5,6$ \\
HD 144432 & A9IVev & 7350 & 32 & $145 \pm 20^{1}$ & 0.05 & $6.5 \pm 0.5$ & II & $3,5,6$ \\
HD 163296 & A3Ve & 8720 & 30 & $122 \pm 15$ & 0.03 & $6.6 \pm 0.4$ & II & $2,5,6$ \\
HD 179218 & B9e & 10500 & 80 & $244 \pm 55$ & 0.17 & $5.0 \pm 0.6$ & I & $2,5,6$ \\
KK Oph & A6 & 8000 & 20 & $160 \pm 30^{1}$ & 0.59 & $6.5 \pm 0.5$ & II & 4,6 \\
51 Oph & A0II/IIIe & 10100 & 289 & $131 \pm 15$ & 0.05 & $5.5 \pm 0.2$ & II & $2,5,6,7$ \\
\hline
\end{tabular}

${ }^{1}$ Error estimated; ${ }^{2}$ Hipparcos parallax; ${ }^{3}$ Pérez et al. (2004); ${ }^{4}$ Hillenbrand et al. (1992); ${ }^{5}$ van den Ancker et al. (1998) and references therein; ${ }^{6}$ Dominik et al. (2003); ${ }^{7}$ van den Ancker et al. (2001).

power spectra of all scans with a white-light fringe amplitude higher than this threshold. To correct for the bias introduced by signal fluctuations not related to the fringe signal, the offfringe power spectrum, determined on the source, but far from zero OPD, is subtracted from the signal. The fringe amplitude (or correlated flux) as function of wavelength is the square root of the fringe power spectra after this "background" subtraction. Division of this final fringe amplitude by the photometric flux gives the raw (instrumental) visibility of the object as function of wavelength. To allow at least approximately for the influence of unequal fluxes in the two interfering beams, the flux for the transformation to visibilities is calculated by the expression $\sqrt{A B}$, where $A$ and $B$ are the fluxes determined from the photometric datasets of the two incoming telescope beams. A precise correction, which would imply determining the fluxes in the two beams for each individual interferometric scan or even each exposed frame, is not possible in the first available measuring mode described here. Calibrated visibilities for the object are obtained by dividing its raw visibility by the instrumental visibility derived for a calibrator star of known diameter (see Table 3), allowing for eventual deviation of the calibrator's known visibility from the point source value 1.0. We are confident that these results are free of spurious fringe detections: overresolved sources do not show traces of a fringe signal. Even for the bright source $\mathrm{OH} 26.5+0.6$ with a $N$ band flux at the time of our observations of $\approx 650 \mathrm{Jy}$ no fringe signal was detectable.

This method of power spectrum analysis has its strength for reasonably bright sources (like the Herbig Ae/Be stars studied in this paper) and then should be comparatively insensitive to atmospheric fluctuations.

A second method, based on coherent integration, which involves a clever kind of shift-and-add has been developed in parallel and will be described elsewhere. It promises to work more reliably for sources with low flux levels. Here, it has been used to independently verify the visibilities obtained from power spectrum analysis.

The errors on the observed visibilities are mostly systematic. The statistical signal-to-noise ratio on the white light fringe amplitudes - if self-fringe tracking is possible at all is $5-10$ at minimum and much better after adding up the several hundred scans taken per interferometric measurement.
The main systematic effect is the varying overlap between the interfering beams due to imperfect source acquisition and residual image motion. This may reduce the fringe signal by different, unknown amounts for object and calibrator measurement, and the same is true for seeing variations. Comparing the raw visibilities observed for different calibrator stars during one night, the standard deviation of these values under good conditions amounts to $\pm 5-10 \%$ (relative) at the red and blue end of the spectrum, respectively, while for adverse conditions these numbers have to be multiplied by a factor of 2-2.5. The visibilitity observed on calibrators ("instrumental visibility") rises from about 0.4 at $8 \mu \mathrm{m}$ to about 0.7 at $13 \mu \mathrm{m}$, rather repeatable from night to night.

\section{The sample}

The core of our small sample consists of five isolated Herbig Ae stars for which near- to mid-infrared spectra were available from the ISO spectrometer SWS. It includes both sources with strong and with moderate mid-infrared excess, and these sources showed the emission signatures corresponding to the presence of different dust populations in their circumstellar environment. With KK Oph and 51 Oph one source more closely associated to molecular cloud material and one source with rather optically thin circumstellar environment, respectively, were added to cover a wider range of Herbig Ae/Be stars. None of these stars has a known close (i.e. sub-arcsec) companion, so that the mid-infrared excesses should be solely due to diffuse emission. Unknown companions with separations of 80 mas or more would have shown clearly in our visibility measurements by several cycles of the sinusoidal variation typical for binary sources.

\subsection{Description of the sample}

In Table 1 we summarize some basic properties of the seven Herbig Ae/Be stars studied in this paper. The stars cover a fairly narrow range in temperature and - except for $51 \mathrm{Oph}-$ in luminosity, thus allowing meaningful comparisons of other properties. The stars are not embedded in their parental molecular cloud; the bulk of the infrared emission seen with large aperture telescopes (e.g. IRAS and ISO) should therefore be due to their circumstellar disks, even in the case of KK Oph. Figure 1 
Table 2. Journal of observations.

\begin{tabular}{|c|c|c|c|c|c|}
\hline \multirow[t]{2}{*}{ Object } & \multirow{2}{*}{$\begin{array}{c}10 \mu \mathrm{m} \text { flux } \\
(\mathrm{Jy})\end{array}$} & \multirow{2}{*}{$\begin{array}{l}\text { UT date } \\
\text { (2003) }\end{array}$} & \multicolumn{2}{|c|}{ Projected baseline } & \multirow{2}{*}{$\begin{array}{l}\text { Nb. of point sources } \\
\text { Resp. names of calibrators }\end{array}$} \\
\hline & & & Length (m) & $\mathrm{PA}\left({ }^{\circ}\right)$ & \\
\hline HD 100546 & $65.8^{i}$ & 17.6. $0 \mathrm{~h}$ & 74.4 & 60.5 & HD 89388, HD 152161 \\
\hline HD 142527 & $10.4^{i}$ & 14.6. $0 \mathrm{~h}$ & 102.0 & 11 & HD $120323^{a}$, HD $120323^{b}$, HD 168454 \\
\hline HD 144432 & $7.5^{i}$ & 17.6. $2 \mathrm{~h}$ & 102.0 & 26.2 & HD 89388, HD 152161, HD $139063^{a}$, HD $139063^{b}$ \\
\hline HD 144432 & $7.5^{i}$ & 17.6. $5 \mathrm{~h}$ & 97.0 & 42.5 & HD $139063^{a}$, HD $139063^{b}$, HD 139997 \\
\hline HD 163296 & $18.2^{i}$ & 14.6. $3 \mathrm{~h}$ & 99.3 & 16 & HD $167618^{a}$, HD $167618 b^{b}$ \\
\hline HD 179218 & $23.4^{i}$ & 16.6. $3 \mathrm{~h}$ & 59.7 & 12.9 & HD $165135^{b}$, HD $187642^{a}$, HD 152161, HD $187642^{b}$, HD 167618 \\
\hline HD 179218 & $23.4^{i}$ & 16.6. $6 \mathrm{~h}$ & 86.0 & 40.0 & HD $165135^{b}$, HD $187642^{a}$, HD 152161, HD $187642^{b}$, HD 167618 \\
\hline KK Oph & 11.3 & 17.6. $1 \mathrm{~h}$ & 100.3 & 8.8 & HD 152161, HD $139063^{a}$ \\
\hline KK Oph & 11.3 & 17.6. $4 \mathrm{~h}$ & 102.4 & 28 & HD $139063^{a}$, HD $139063^{b}$ \\
\hline $51 \mathrm{Oph}$ & $15.7^{i}$ & 15.6. $3 \mathrm{~h}$ & 101.2 & 23 & HD $168454^{a}$, HD $168454^{b}$, HD 167618 \\
\hline $51 \mathrm{Oph}$ & $15.7^{i}$ & 15.6. $7 \mathrm{~h}$ & 101.4 & 38 & HD $168454^{a}$, HD $168454^{b}$, HD 167618 \\
\hline $51 \mathrm{Oph}$ & $15.7^{i}$ & 15.6. $8 \mathrm{~h}$ & 85.6 & 45 & HD $168454^{a}$, HD $168454^{b}$, HD 167618 \\
\hline $51 \mathrm{Oph}$ & $15.7^{i}$ & 16.6. $0 \mathrm{~h}$ & 98.8 & -7 & HD $165135^{a}$, HD 152786, HD $165135^{b}$ \\
\hline $51 \mathrm{Oph}$ & $15.7^{i}$ & 16.6. $2 \mathrm{~h}$ & 99.6 & 14 & HD $165135^{a}$, HD 152786, HD $165135^{b}$ \\
\hline
\end{tabular}

${ }^{a}$ First of several measurements of the calibrator during this night, the others are designated ${ }^{b},{ }^{c} ;{ }^{i}$ IRAS $12 \mu$ m flux.

Table 3. Parameters of calibrator stars.

\begin{tabular}{lcrcc}
\hline \hline Calibrator & $\begin{array}{c}\text { Spectral } \\
\text { type }\end{array}$ & $\begin{array}{r}\text { Flux } \\
(\mathrm{Jy})\end{array}$ & $\begin{array}{c}\text { Uniform disk } \\
\text { diameter (mas) }\end{array}$ & Ref. \\
\hline HD 89388 & K3IIa & $33.2^{i}$ & $5.17 \pm 0.10$ & 2 \\
HD 120323 & M4.5III & $176^{i}$ & $9.16 \pm 0.07$ & 2 \\
HD 139063 & K5III & $30.1^{i}$ & $4.47 \pm 0.24$ & 1 \\
HD 139997 & K5III & $14.5^{i}$ & $3.46 \pm 0.38$ & 2 \\
HD 152161 & M3II-III & $35.25^{i}$ & $4.83 \pm 0.50$ & 2 \\
HD 152786 & K3III & $82.2^{i}$ & $7.21 \pm 0.21$ & 2 \\
HD 165135 & K0III & $16.3^{i}$ & $3.33 \pm 0.05$ & 2 \\
HD 167618 & M3.5III & $149^{i}$ & $11.33 \pm 0.04$ & 2 \\
HD 168454 & K3IIIa & $43.7^{i}$ & $5.78 \pm 0.15$ & 2 \\
HD 187642 & A7V & $23.2^{i}$ & $3.22 \pm 0.01$ & 2 \\
\hline
\end{tabular}

${ }^{1}$ CHARM catalogue, Richichi \& Percheron (2002).

${ }^{2}$ Catalogue of MIDI calibrators, in preparation for publication.

${ }^{i}$ IRAS $12.5 \mu$ m flux.

shows the visual to millimeter spectral energy distributions of the programme stars.

The data are taken from de Winter et al. (2001) and references therein with exception of the $1.3 \mathrm{~mm}$ point of KK Oph, which was taken from Henning et al. (1994).

The solid lines in Fig. 1 show the best fit DDN models for our programme stars, taken from Dominik et al. (2003) for all stars except KK Oph, where the model was done for this paper, and 51 Oph. We will return to these stars in Sects. 5.2 and 5.3.

\subsection{Notes on individual objects}

HD 100546 The infrared spectrum of this group I source is characterized by strong emission from crystalline forsterite $\left(\mathrm{Mg}_{2} \mathrm{SiO}_{4}\right.$, see Malfait et al. 1998a), that seems to be located at a typical distance of $\sim 10 \mathrm{AU}$ from the star (Bouwman et al. 2003). The PAH emission is strong and spatially resolved on a scale of $\sim 100 \mathrm{AU}$ (van Boekel et al. 2004). The $10 \mu \mathrm{m}$ and $20 \mu \mathrm{m}$ emission was recently shown resolved (Liu et al. 2003) on a scale of $\approx 24 \mathrm{AU}$.
HD 142527 The $10 \mu \mathrm{m}$ spectrum of HD 142527 shows a flattopped silicate emission feature with an excess at $11.3 \mu \mathrm{m}$ indicating grain growth and crystallisation of the warm silicate grains in the disk atmosphere (Bouwman et al. 2001). This source was originally classified by Meeus et al. (2001) as a group I source, on the basis of the strongly rising far-IR spectrum. The optical and IR luminosity being equal, the spectrum of HD 142527 cannot easily be explained in the context of a passive centrally irradiated disk, since in such disks the maximum reprocessed luminosity is half of the stellar luminosity. The lack of substantial veiling in the optical spectrum of HD 142527 precludes active accretion as the source of extra luminosity at IR wavelengths, as does the observed silicate emission at 10 and $20 \mu \mathrm{m}$ (Malfait et al. 1999; Meeus et al. 2001). Ignoring the $40 \mu \mathrm{m}-200 \mu \mathrm{m}$ spectral range, the source should be classified as a group II source. We consider it as such in the remainder of this paper. The excess IR emission beyond 30-40 $\mu \mathrm{m}$ must be due to a close-by source.

HD 144432 The $10 \mu \mathrm{m}$ silicate band of HD 144432 is one of the strongest observed in HAe stars (van Boekel et al. 2003) and has a shape that closely resembles that of the interstellar medium. The IR spectrum falls steeply which implies a group II classification.

HD 163296 The millimeter continuum and CO ( $J=1-0)$ rotational line emission of this group II source was spatially resolved by Mannings \& Sargent (1997), providing strong evidence for the presence of a disk. The millimeter slope of $S_{v} \propto$ $v^{2}$ suggests that the cold mid-plane grains are millimeter-sized (assuming the disk is optically thin at these wavelengths). The ISO spectra show only weak evidence for crystalline silicates (Bouwman et al. 2001). A broad $20 \mu \mathrm{m}$ band centered at $23 \mu \mathrm{m}$ was identified with FeS by Keller et al. (2002). Clearly, the dust in the disk of HD 163296 is already substantially modified from the ISM dust composition.

HD 179218 Strong PAH emission bands dominate the $10 \mu \mathrm{m}$ spectrum of HD 179218. The $10 \mu \mathrm{m}$ silicate emission band as well as the ISO spectrum (Meeus et al. 2001) show that crystalline dust is abundant in this group I star. While the crystalline 


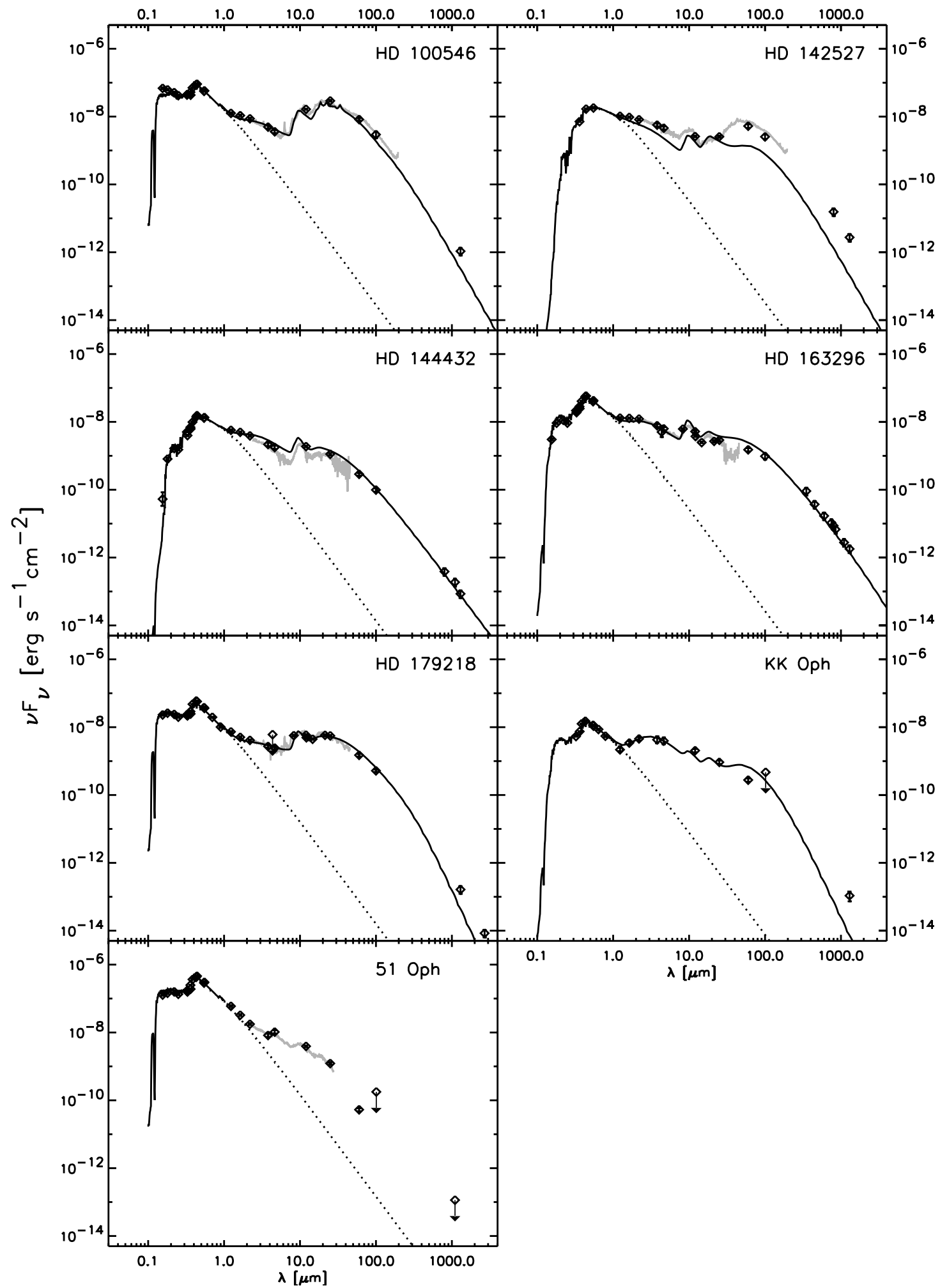

Fig. 1. Observed visual to millimeter spectral energy distributions of the programme stars. Dereddened fluxes, taken from the literature, are shown as diamonds, while the curves in grey give SWS and LWS spectra from the infrared satellite ISO. The solid line represents the sum of the stellar photosphere (dotted line) and the best fit DDN model, taken from Dominik et al. (2003) for all stars except KK Oph, where a new model was constructed, and $51 \mathrm{Oph}$. For $51 \mathrm{Oph}$ no fit of the spectral energy distribution by a DDN-type disk model was possible (see text).

silicates in HD 100546 are dominated by forsterite, HD 179218 also shows pyroxenes, probably enstatite $\left(\mathrm{MgSiO}_{3}\right.$; Bouwman et al. 2001).
51 Oph The A0 giant 51 Oph has double-peaked $\mathrm{H} \alpha$ emission, suggesting the gas is in a rotating disk (Waters, private communication). The ISO spectrum of 51 Oph is very different 
from that of other HAe stars (van den Ancker et al. 2001) and shows emission from gas-phase molecules $\left(\mathrm{CO}, \mathrm{CO}_{2}, \mathrm{H}_{2} \mathrm{O}\right)$. The $10 \mu \mathrm{m}$ spectrum shows a silicate band, though less strong than the other sources in our sample. The spectrum drops steeply longwards of $25 \mu \mathrm{m}$. Millimeter emission from cold grains has so far not been detected. Both observations suggest that 51 Oph does not have a substantial reservoir of cold grains. A close companion which could have truncated the circumstellar dust distribution and thus removed the colder material is not known. Clearly the nature of the disk in this group II star is very different from that in other HAe stars.

KK Oph This is a PMS binary (Leinert et al. 1997) with a separation between both components of 1.6". The star shows UX Ori type variations (Herbst \& Shevchenko 1999) which probably indicates that we see the disk at a rather inclined angle (Natta et al. 2000; Dullemond et al. 2003). The latter authors also suggest that UX Ori stars have self-shadowed disks. Consistently, the scheme of van Boekel et al. (2003) classifies the star as a group II source. The DDN fit plotted in Fig. 1 is new and was done with the following parameters: $T_{\text {eff }}=$ $8700 \mathrm{~K}, L=20 L_{\odot}, M=2.0 M_{\odot}, \mathrm{SpT}=\mathrm{A} 7, d=165 \mathrm{pc}$, $T_{\text {rim }}=1200 \mathrm{~K}$, vertical expansion factor at inner rim $\chi_{\text {rim }}=$ $4.0, M_{\text {disk }}=0.02 M_{\odot}$, exponent of density law $=-2.7, r_{\text {out }}=$ $200 \mathrm{AU}, i=70^{\circ}$.

\section{Results}

The observing sequence for our interferometric measurements described above implies that in principle three types of data are available for each observed source: single-dish acquisition images at $8.7 \mu \mathrm{m}(F W H M 1.4 \mu \mathrm{m})$, single dish low resolution $(\lambda / \Delta \lambda \approx 30)$ spectra over the full $N$ band $(7.5 \mu \mathrm{m}-13.5 \mu \mathrm{m})$, and the spectrally resolved interferometric measurements of correlated flux, respectively the visibility derived from it.

\subsection{Imaging}

The $8.7 \mu \mathrm{m}$ single dish acquisition images are a valuable supplement to the interferometric measurements. They provide low spatial frequency information on the sources which by design is not contained in the interferometric measurements. These images are used after the pointing to test if the target is within the MIDI FOV (diameter of about $3^{\prime \prime}$ ) and to perform a fine pointing. Chopping $\left(f=2 \mathrm{~Hz}\right.$, angle $=-90^{\circ}$, amplitude $=$ $\left.10^{\prime \prime}\right)$ is needed to visualize the star, which is not perfectly centered in the first image, and centered in a second step. The number of frames recorded per image was 2000 and the exposure time is by default limited to $4 \mathrm{~ms}$ in order to avoid background saturation. The frame cycle rate is close to $10 \mathrm{~ms}$, so the recording of an image lasts about $20 \mathrm{~s}$.

Normally these images are unresolved or at most barely resolved for our sources, with the notable exception of HD 100546. Figure 3 shows a contour map of this source after deconvolution with the point spread function seen on the interferometric calibrator star HR 6257 (HD 152161). The pixel size on the sky was 98 mas. This scale factor has been derived from observations of close visual binaries. The deconvolution has been performed using the Lucy-Richardson algorithm (1974) with 40 iterations. This number is sufficient to increase the spatial resolution of the image and to provide good convergence. The deconvolved image immediately shows that in this source the warm dust is also distributed in a flattened, most probably disk-like geometry. A two-dimensional Gaussian fit to the brightness distribution gives a $F W H M$ along the long and short axes of $283 \pm 34$ mas and $180 \pm 84$ mas (29 AU and 19 AU, respectively). This agrees with the size of $\sim 24$ AU found by Liu et al. (2003) by nulling interferometry, but is a much more direct measurement. The position angle of the long axis is $134 \pm 5^{\circ}$, essentially the same as the $127 \pm 5^{\circ}$ found by Grady et al. (2001) on arcsec scales. The disk-like distribution of circumstellar matter appears to be continuous at least down to the 100 mas (10 $\mathrm{AU}$ ) range.

The fact that HD 100546 is resolved by a single UT telescope with a FWHM similar to that of an Airy disk does not mean that it is pointless to derive and discuss its visibilities. Of course, the resulting visibility values will be small (see Fig. 5).

\subsection{8-13 $\mu m$ spectra}

Figure 2 shows the low-resolution $N$ band spectra obtained with MIDI for the sources of our sample during the spectrophotometric observations needed for the determination of visibilities from the correlated fluxes. These spectra result from chopped observations at $2 \mathrm{~Hz}$ with a stroke of $10^{\prime \prime}$. The typical on-source exposure time is $20 \mathrm{~s}$. Spectra obtained for the same sources with TIMMI2 on the $3.6 \mathrm{~m}$ telescope on La Silla are overplotted. The generally good agreement gives us confidence in the calibration and operation of MIDI. The errors in the shape of the spectra are $5 \%$ to $10 \%$, in the absolute value $10 \%$ to $15 \%$.

All of the spectra show silicate in emission, as expected for circumstellar disks where we see the heated surface layers. For sources with strong variability, the essentially simultaneously obtained spectra will have the value to show the status of the object to which the interferometric measurements refer.

\subsection{Visibilities}

In Fig. 4 we show the visibilities as a function of wavelength (which at the same time means with decreasing spatial frequency) as observed with MIDI. The errors give the standard deviation obtained by reducing the object data sets with different calibrator stars observed during the same night, a representative measure of the uncertainty in the visibility values. Most programme stars show a remarkably similar pattern with a moderately high visibility near $8 \mu \mathrm{m}$, followed by a notable drop between $8 \mu \mathrm{m}$ and $9 \mu \mathrm{m}$ and roughly constant values beyond. There is no obvious structure in the visibility curves related to the silicate emission feature. This shape appears typical. It reflects the distribution of the emitting material over a range of temperatures with distance, with a particular concentration of the warmest material at small distance from the star. The objects 51 Oph and HD 179218 are special in this respect, and this will be discussed below in Sect. 5.2. Figure 4 also includes, for comparison and subsequent discussion, predicted 

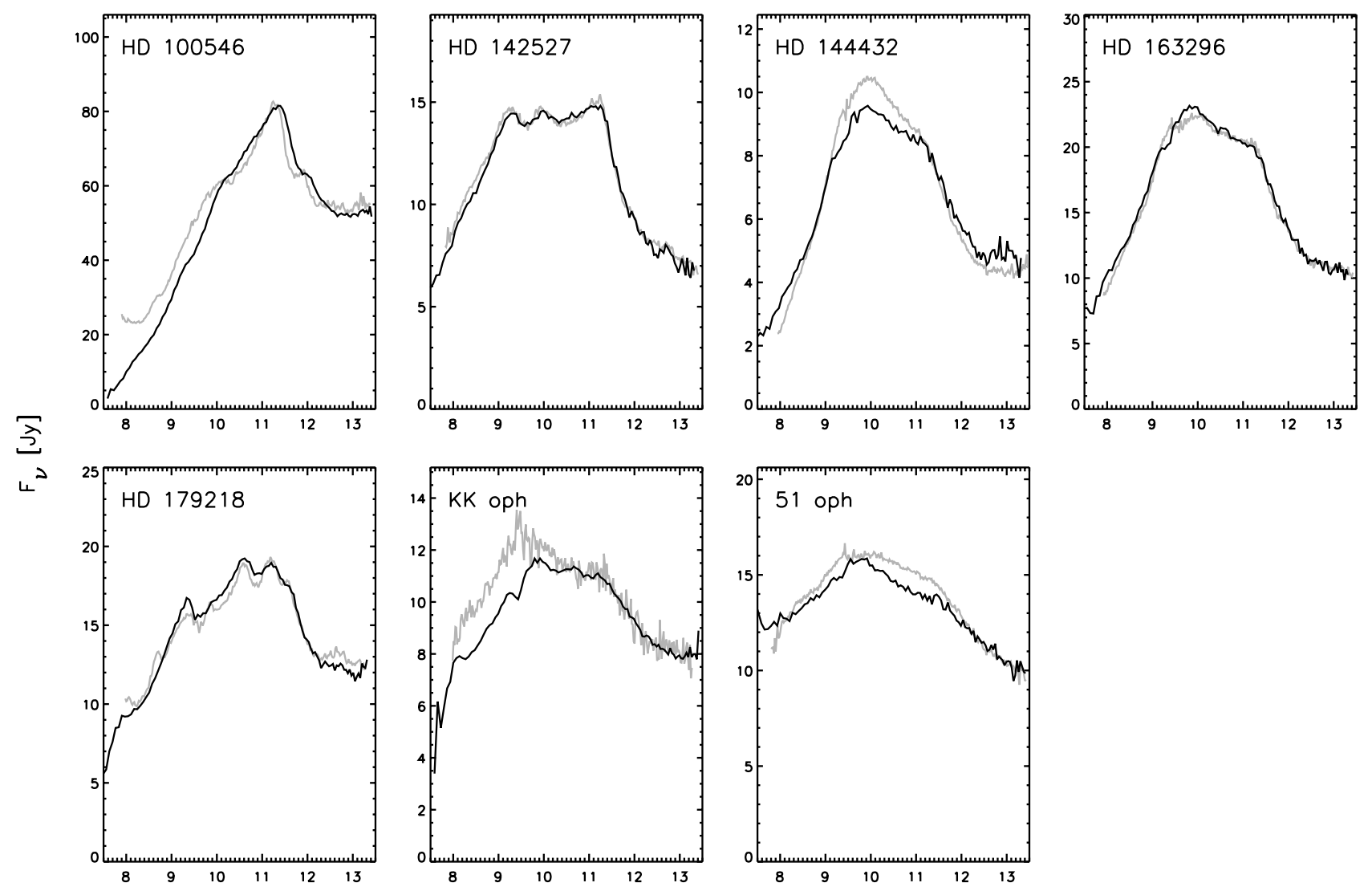

$\lambda[\mu \mathrm{m}]$

Fig. 2. Observed 7.5-13.5 $\mu \mathrm{m}$ spectra of the programme stars. Note the large range in silicate band shapes, and the presence of crystalline silicates (shoulder at $11.3 \mu \mathrm{m}$ ) in some objects. PAH bands at 7.9, 8.6 and $11.3 \mu \mathrm{m}$ are prominent in HD 100546 and HD 179218 . The MIDI spectra are indicated in black. TIMMI 2 spectra are drawn in grey. In order to allow a good comparison of the spectral shapes, scaling factors (from top left to bottom right: $\times 1.08, \times 1.15, \times 1.25, \times 0.92, \times 0.75, \times 0.90, \times 1.05$ ) have been applied to the TIMMI 2 spectra. Given the fact that the absolute level of the TIMMI2 data was simply determined by adjusting to IRAS results, the level of agreement in shape and absolute value between these fully independent sets of spectra gives confidence to both.

visibilities based on DDN models of passive, centrally irradiated circumstellar disks. These models were constructed with the sole purpose to fit the spectral energy distribution of the individual objects without any spatial constraint except the radius of sublimation around the central star. Therefore they are not necessarily expected to provide a good representation of the observed visibilities.

For 51 Oph no model prediction is shown. This source cannot be described by a DDN model, since its environment violates one of the physical assumptions of that model: optical thickness of the circumstellar disk out to the mid-infrared range. Instead, for $51 \mathrm{Oph}$, we restrict ourselves to estimating a size by comparing to the simplest geometrical ad hoc model we can think of, a Gaussian brightness distribution. This is a reasonable approximation for barely resolved (high visibility) objects. The visibility of such a distribution is calculated as

$V(f)=V_{0} \exp \left(-3.56 f^{2} \Theta^{2}\right)$,

where $\Theta$ is the FWHM (") of the Gaussian distribution and $f$ the spatial frequency in units $\operatorname{arcsec}^{-1}$. The results are given in Table 4 and discussed together with the mid-infrared size estimates for the other sources below.

\section{Discussion}

The results presented in this paper refer to interferometric observations with only one or a few similar baseline settings. For dedicated discussions of individual objects we have to await complementing observations on different baselines or to study in detail the behaviour of the spectra on different spatial scales. Here we concentrate on the general view on the geometrical size of the circumstellar dust distribution around Herbig Ae/Be stars, as given by these new observations for the $10 \mu \mathrm{m}$ wavelength region.

\subsection{Size of the emitting regions}

All of the sources were resolved with MIDI in the $10 \mu \mathrm{m}$ range over the projected baselines of $60 \mathrm{~m}-100 \mathrm{~m}$ resulting during the observing run. This means that the $10 \mu \mathrm{m}$ sizes of the observed disks are larger than 3 mas $F W H M$. We take this as detection limit since at the short wavelength end of the $10 \mu \mathrm{m}$ band it will reduce a visibility by more than $10 \%$ from the point source value of 1.0. 
Table 4. Sizes of the emitting regions ${ }^{1}$.

\begin{tabular}{lccccc}
\hline \hline Object & $\begin{array}{c}\text { SED } \\
\text { group } \\
\text { association }\end{array}$ & $\begin{array}{c}\text { Average } \\
\text { visibility } \\
(9-13 \mu \mathrm{m})\end{array}$ & $\begin{array}{c}\text { Radial } \\
\text { surface density } \\
\text { gradient }\end{array}$ & $\begin{array}{c}\text { Half-light } \\
\text { radius (") } \\
\text { at 12.5 } \mu \mathrm{m}\end{array}$ & $\begin{array}{c}\text { Half-light } \\
\text { radius (AU) } \\
\text { at } 12.5 \mu \mathrm{m}\end{array}$ \\
\hline HD 100546 & I & $0.040 \pm 0.008$ & 0.04 & $0.050 \pm 3 \% \%^{2}$ & $5.1 \pm 0.5^{3}$ \\
HD 142527 & II & $0.249 \pm 0.055$ & -0.52 & $0.011 \pm 5 \%^{2}$ & $2.1 \pm 0.2^{3}$ \\
HD 144432 & II & $0.183 \pm 0.021$ & -0.30 & $0.014 \pm 3 \%^{2}$ & $2.1 \pm 0.2^{3}$ \\
HD 163296 & II & $0.203 \pm 0.017$ & -1.18 & $0.007 \pm 2 \% \%^{2}$ & $0.8 \pm 0.1^{3}$ \\
HD 179218 & I & $0.091 \pm 0.014$ & 0.21 & $0.034 \pm 3 \%^{2}$ & $8.2 \pm 0.8^{3}$ \\
KK Oph & II & $0.313 \pm 0.037$ & -0.57 & $0.010 \pm 5 \%^{2}$ & $1.6 \pm 0.2^{3}$ \\
51 Oph & II & $0.643 \pm 0.063$ & - & $0.007 \pm 8 \% \%^{3}$ & $0.5 \pm 0.07^{4}$ \\
\hline
\end{tabular}

${ }^{1}$ Derived from optically thin power law radial density distributions (see text).

${ }^{2}$ Error from visibility uncertainty.

${ }^{3}$ Modelling uncertainty of $10 \%$ assumed.

${ }^{4}$ Half of the $F W H M$ of a Gaussian distribution giving the observed visibility at $12.5 \mu \mathrm{m}$.

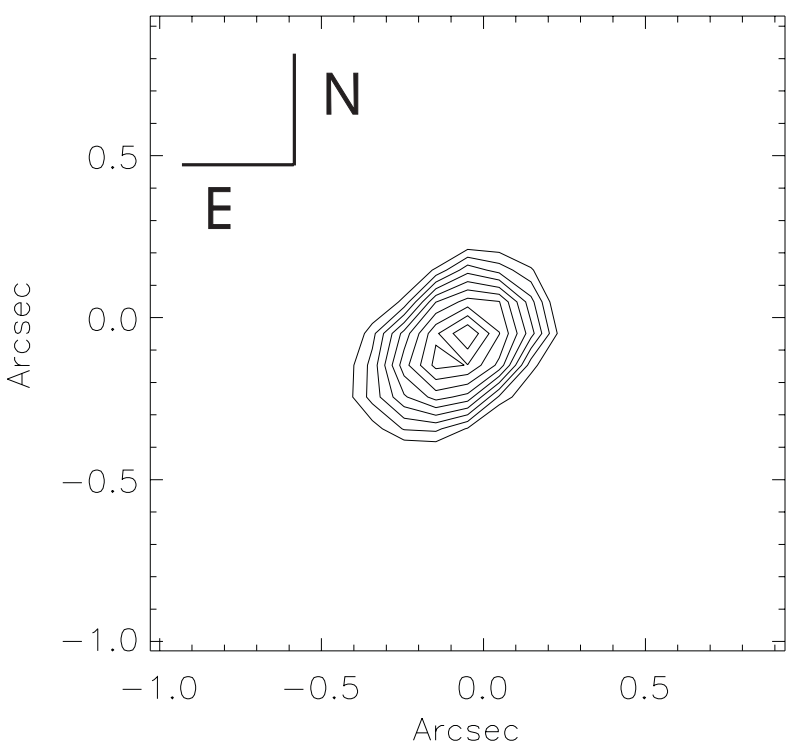

Fig. 3. Isophotal representation of the deconvolved $8.7 \mu \mathrm{m}$ image of HD 100546. The contour levels are linearly spaced from $100 \%$ to $25 \%$ for the fourth root of the image brightness $I$, i.e. they are $\propto I^{1 / 4}$. The last contour therefore is equivalent to $0.4 \%$ of the maximum of $I$. The orientation of the $0.5^{\prime \prime}$ slit during the exposure was at position angle $140^{\circ}$, i.e. along the long axis of the object.

To derive realistic size estimates for our sample of typically well resolved $\mathrm{Ae} / \mathrm{Be}$ stars, Gaussian fits are not appropriate since they have too steep a brightness decrease with distance from the star with respect to the more gradual light distribution in a circumstellar disk. Instead, we choose to use a simple model thought to approximate this light distribution in a better way. Outside of the sublimation radius $r_{0}$ we assume an optically thin distribution of grey particles with the radial distribution of their surface density given by a power law

$\Sigma(r)=\Sigma_{0}\left(r / r_{0}\right)^{-m}$

as typically used in circumstellar disk models. The equilibrium temperature of such a distribution is given by

$T(r)=T_{0}\left(r / r_{0}\right)^{-1 / 2}$ where again $r_{0}$ is the radius at which for grey particles the sublimation temperature $T_{0}=1500 \mathrm{~K}$ is reached. The exponent of the power law for the spatial distribution is the free variable which can be varied to reproduce the observed visibility. We choose to fit the visibility value at $12.5 \mu \mathrm{m}$, since this wavelength is practically free of silicate emission. The resulting angular and linear sizes, given as radius encircling half of the total emission at this wavelength, are shown in Table 4. The radii fall into the range of $1 \mathrm{AU}$ to $10 \mathrm{AU}$, which happens to be not too far from the $\approx 3.5 \mathrm{AU}$ at which grey particles would have their maximum emission at this wavelength for a luminosity of $40 L_{\odot}$, typical for our sample stars. Since our mid-infrared sizes were measured in a reasonably uniform way, we can compare them with other properties of the sources.

\subsection{Spatial distribution of mid-infrared emission}

In Fig. 5 we have plotted the sizes shown in Table 4 as function of the mid-infrared spectral slope represented by the IRAS colour between $12 \mu \mathrm{m}$ and $25 \mu \mathrm{m}$, $-2.5 \log \left(F_{v}(12 \mu \mathrm{m}) / F_{v}(25 \mu \mathrm{m})\right.$. A correction has been applied to the mid-infrared colour of HD 142527, indicated by the arrow in Fig. 5. We recall that the excess emission beyond 30$40 \mu \mathrm{m}$ in this object, well represented by blackbody emission of $\approx 70 \mathrm{~K}$, probably is due to a nearby very red source. This means that up to $60 \%$ of the $25 \mu \mathrm{m}$ flux could be due to such contamination, and we choose to correct the IRAS colour of HD142527 according to half of this value to be conservative. With this in mind, we see within our small sample a correlation between estimated size and the gradient of the mid-infrared SED, in the sense that the largest objects (which also are those with the lowest measured visibility) have the reddest colours.

This correlation, if generally confirmed for Herbig Ae stars, would give the phenomenological classification into sources of group I and group II by Meeus et al. (2001) an observable physical foundation and also give some support to their interpretation in terms of flaring versus non-flaring disks. It also would give some support to their explanation of this effect: the reddest objects (group I) are observed to be larger, i.e. their mid-infrared radiation is spread out to larger distances. This 


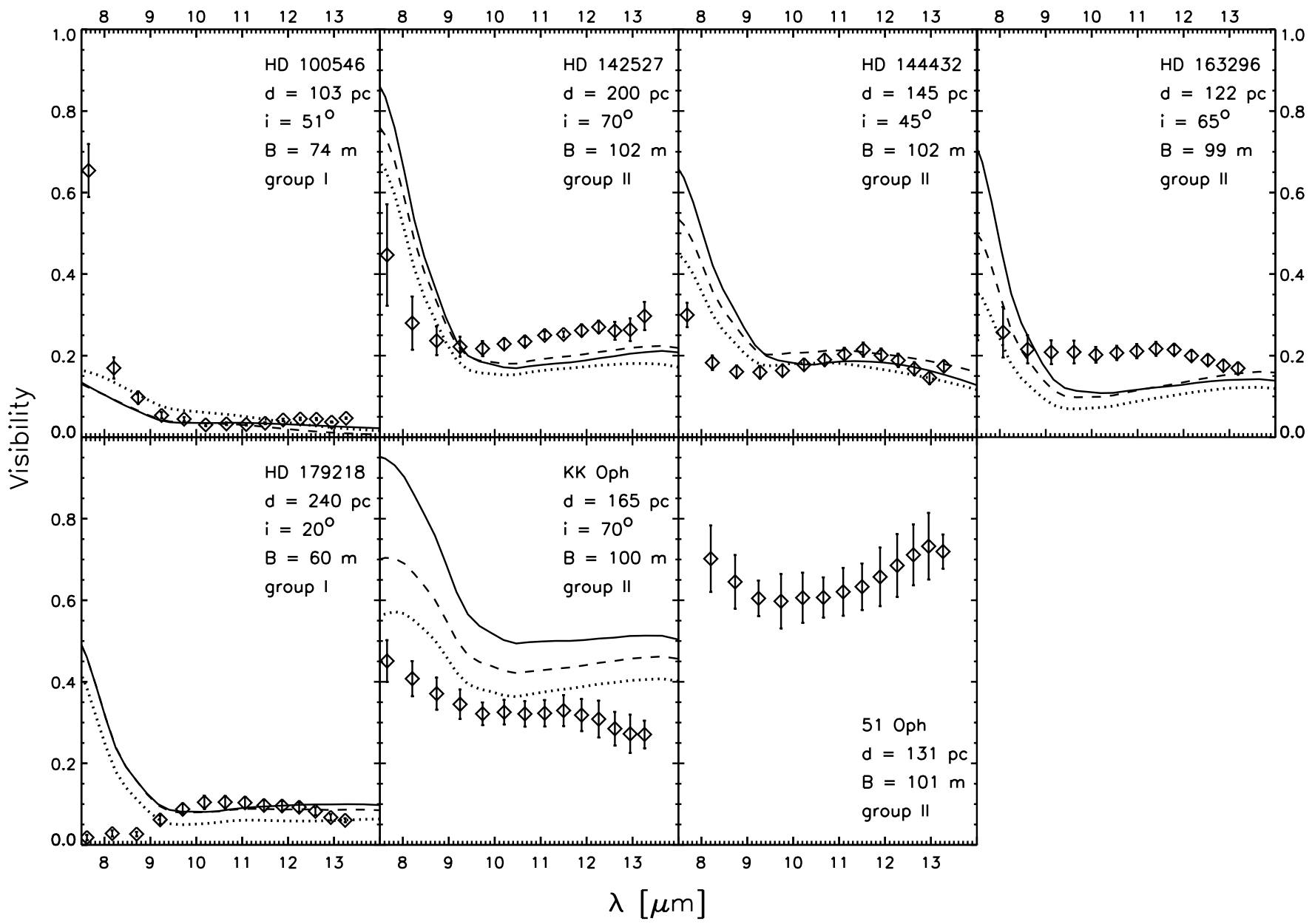

Fig. 4. The observed visibilities of the programme stars with the model predictions overplotted. These models were made with the sole purpose of fitting the spectral energy distribution and do not contain any feedback from the visibility observations. Three model visibility curves are shown: two referring to the inclination used in the SED fitting and calculated for a cut along the long axis (broken line) and along the short axis (solid line) respectively, while the dotted line is the prediction for a pole-on view. The errors give the standard deviation of the visibility results obtained for one source with different calibrators.

naturally can be explained by a flaring disk geometry which would expose more distant material to direct illumination by the central star.

With some caution, we can repeat the argument, but now based on the difference in spectrally resolved visibility between the most typical group I source HD 100546 and the group II sources of our sample (Fig. 4). While near $8 \mu \mathrm{m}$ the visibilities of all sources are roughly similar, the group I source HD 100546 shows a much more pronounced drop in visibility with wavelength compared to the group II sources between $8 \mu \mathrm{m}$ and $9 \mu \mathrm{m}$, and beyond $9 \mu \mathrm{m}$ has substantially lower visibility than the group II sources. It means that the $10 \mu \mathrm{m}$ emitting dust is systematically farther away from the star in this group I source. This is in qualitative agreement with the concept that group I sources have flaring disks, better exposed to stellar radiation still at larger distances, and group II sources do not. We may have found an access to "see" the gross features of the disk geometry in the inner circumstellar regions.

For different reasons, 51 Oph and HD 179218 do not fit well this otherwise convincing interpretation of visibilities. For $51 \mathrm{Oph}$, as already mentioned, the disk is much more compact than that of the other stars. This can also be seen from the different shape of the IR spectrum and the lack of any detected millimeter continuum emission. In consequence the visibility values are consistently much higher than for the other group II sources. For HD 179218, the second group I source in our sample, the low visibility values also point to warm emitting dust at larger distances from the star than in the group II sources. However, the very low visibility seen around $8 \mu \mathrm{m}$ indicates a special, possibly double-peaked, spatial structure for this emission. Conceivably also the PAH emission, particularly strong in this source, could have lowered the resulting visibility value at the shortest wavelengths.

\subsection{Comparison to model predictions}

For simplicity we limit ourselves here to the existing DDN set of models that fit the SEDs of our programme stars. Adding other sets of models would imply evaluating the relative merits of these approaches. This should be done, and carefully, but will be a paper of its own. 
12.5 micron

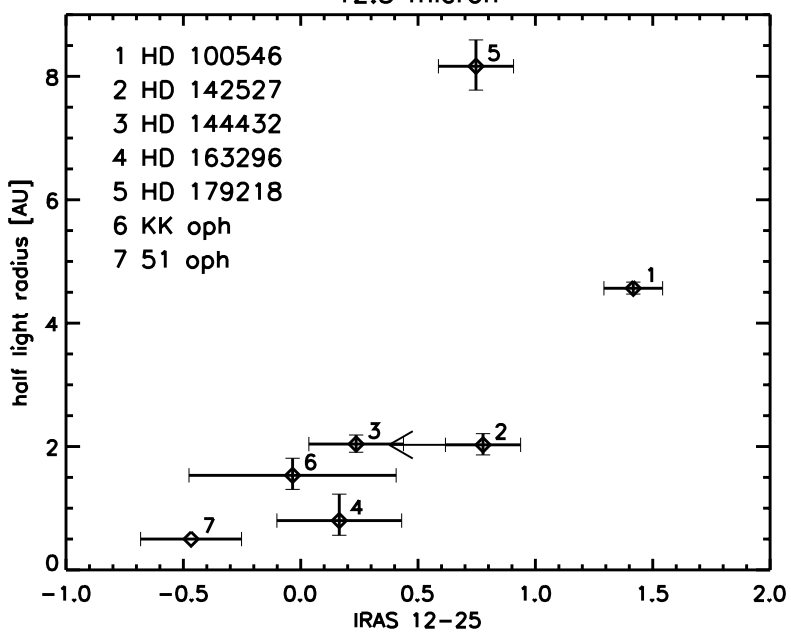

Fig. 5. Correlation between the mid-infrared spectral slope (taken from IRAS as $-2.5 \log \left(F_{v}(12 \mu \mathrm{m}) / F_{v}(25 \mu \mathrm{m})\right)$ and the half-light radius corresponding to the observed visibilities at $12.5 \mu \mathrm{m}$. The errors reflect the uncertainty in IRAS fluxes and visibility measurements; the resulting uncertainty in size may be optimistic. The largest sources are those with the reddest mid-infrared spectral distribution, and these were also classified as group I. For HD 142527 the arrow shows the correction for contamination of the IRAS colour by an unrelated very red nearby source (see text).

\subsubsection{Description of the models}

The DDN models refer to passive, centrally irradiated circumstellar disks with an inner hole (Dullemond et al. 2001). The SED emerging from such a disk primarily has two main components: (i) an optically thin emission from the surface layer, responsible for the observed solid-state emission features and part of the near-and mid-IR flux, and (ii) a component originating from the midplane of the disk which contributes to the mid-IR flux and dominates the far-IR and sub-millimeter wavelength regions. In the innermost regions of Herbig Ae/Be disks, the temperatures become so high that dust grains evaporate. At the interface between dusty and dust free regions, a puffed-up inner rim forms. It intercepts up to $25 \%$ of the stellar radiation and re-emits as a blackbody component of typically 1200 $1500 \mathrm{~K}$. Just outside the inner rim, a shadow is cast over the disk out to a radius of about 5-10 AU. The hot inner rim as third component in the SED, effective in explaining the nearinfrared $(2-7 \mu \mathrm{m})$ bumps observed in some Herbig Ae/Be stars, is the essential new ingredient in the DDN models with respect to earlier approaches (Kenyon \& Hartmann 1987; Chiang \& Goldreich 1997). As in these older models, a flaring disk geometry in which the surface curves upwards as a function of distance from the star is normally used.

With suitable parameters, these DDN models provide a reasonable fit to the SEDs (star plus disk) of most Herbig Ae stars, (Dominik et al. 2003), and they were constructed just for this purpose. No spatial information has entered into the fitting procedure. The fundamental stellar parameters are taken from Meeus et al. (2001), while the disk is described by the parameters disk mass $M_{\text {disk }}$, the slope of the surface density power law $p$, the outer disk radius $r_{\text {out }}$, the inclination $i$ and in some cases the height of the inner rim $\chi$. Dust grains $0.1 \mu \mathrm{m}$ in size are assumed in a mixture of astronomical silicate (Draine \& Lee 1984) and carbon (Laor \& Draine 1993). The contribution by direct stellar flux is usually negligeable (Fig. 1). For most programme stars, fits were already available (Dominik et al. 2003). For KK Oph, the fit to the spectral energy distribution yielded the parameters summarised in Sect. 3.2.

\subsubsection{Comparison of visibilities}

The general observed trend of visibility with wavelength is given by a pronounced decrease between $8 \mu \mathrm{m}$ and $9 \mu \mathrm{m}$ followed by a plateau out to $13 \mu \mathrm{m}$. This trend appears even clearer in the visibility predictions based on SED fitting DDN models, which also are plotted in Fig. 4. The tentative explanation for this behaviour is that the hot inner rim region of the circumstellar disks gives an overproportional contribution to the shorter wavelength region, resulting in a smaller effective size at these wavelengths than would be expected for the smooth temperature distribution in the disk. We take the general agreement between observations and predictions as qualitative confirmation of the physical picture underlying the DDN models. However, quantitatively there remain significant differences between model predictions and observations to the extent that in no case there is real good agreement between them. The observed decrease in visibility is steeper (for HD 100546) or shallower (for HD 142527, HD 144432, KK Oph) than predicted. The level of the "plateau" longward of $9 \mu \mathrm{m}$ is also different in some cases. But these differences are usually less than $30 \%$ of the observed visibility which will translate to smaller corrections in size because of the non-linear transformation to visibilities. The largest deviation is seen in HD 163296 with a factor of 1.5-1.8 which translates into differences in the width of an equivalent Gaussian distribution of $13 \%$ to $20 \%$, which is not a dramatic effect.

Certainly, one does not expect a perfect spatial distribution of infrared emission predicted by models constructed solely to fit the spectral energy distribution. But the discrepancies show that these models cannot be the last word but will need modifications. It will be important to include spatial constraints such as those given by the presented interferometric observations into the next efforts of circumstellar disk modeling to reach a deeper understanding of structure, physics and evolution of such disks.

We do not try in this paper to produce such improved fits. As the probably best example of a spatially and spectrally selfconsistent model for HL Tau (Men'shchikov et al. 1999) shows, this tends to require an extensive effort, best to be done carefully on an object by object basis.

\section{Summary and conclusion}

Our mid-infrared interferometric study of a sample of seven Herbig Ae/Be stars has shown that

- MIDI on the VLTI is well able to resolve the circumstellar dust structures around Herbig Ae stars in the mid-infrared. 
- One source, HD 100546 is already resolved in our singledish images. Here, it can be directly seen that the inner, warm dust is distributed in a disk-like geometry.

- The characteristic $10 \mu \mathrm{m}$ sizes as derived from the interferometric observations by comparison to optically thin distributions of grey particles are 1-10 AU.

- For our small sample, these sizes correlate with the slope of the mid-infrared spectral energy distribution between $10 \mu \mathrm{m}$ and $25 \mu \mathrm{m}$.

- This gives a physical foundation to the phenomenological classification of Herbig Ae/Be stars into groups by their mid-infrared colour.

- This gives support to the distinction of these groups I and II by flaring-dominated versus non-flaring-dominated circumstellar dust distributions.

- Overall the shape of the observed visibility curves is in qualitative agreement with the shapes resulting for the SED-fitting models of Dullemond et al. (DDN, 2001).

- The measured sizes now put spatial constraints on the disk structure which opens the route to more realistic models reproducing both the spectral energy distribution and the spatial scale.

These first results are based on interferometric size (visibility) determinations only, and additional insight will be gained when studying other aspects of the interferometric data, like asymmetries in the circumstellar dust distribution or detailed spectral shapes as function of spatial scale. The availability of these further options shows that long-baseline mid-infrared interferometry, as offered by MIDI on the VLTI, has the potential to become one of the methods of choice for studying circumstellar dust around young stars.

Acknowledgements. It is a pleasure to thank all those without whom these observations could not have taken place: the technical people of the MIDI team who transformed the sketches and wishes of the astronomers into a working instrument, and the ESO VLTI team working on Paranal observatory or in Garching for their continuing and friendly support during the integration and observations and for providing the impressive interferometric infrastructure of the VLTI. We also want to thank for the necessary and important upper-level support provided through the different phases of the project from all of the involved parties, and in particular to S. Beckwith, I. Appenzeller, H.-W. Rix, A. Quirrenbach, M. Tarenghi and A. van Ardenne. Financial support by the Max-Planck-Gesellschaft, by NOVA, The Netherlands Research School in Astronomy, by NFRA, The Netherlands Foundation for Research in Astronomy, by CNRS, the Centre Nationale de Recherche Scientifique, and by the European Southern Observatory ESO is greatfully acknowledged. The TIMMI2 spectrum of KK Oph was kindly provided by Hendrik Linz.

\section{References}

Bouwman, J., Meeus, G., de Koter, A., et al. 2001, A\&A, 375, 950 Bouwman, J., de Koter, A., Dominik, C., \& Waters, L. B. F. M. 2003, A\&A, 401, 577

Chiang, E. I., \& Goldreich, P. 1997, ApJ, 490, 368

D’Alessio, P., Calvet, N., \& Hartmann, L. 2001, ApJ, 553, 321 de Winter, D., van den Ancker, M. E., Maira, A., et al. 2001, A\&A, 380, 609

Dominik, C., Dullemond, C. P., Waters, L. B. F. M., \& Walch, S. 2003, A\&A, 398, 607

Draine, B. T., \& Lee, H. M. 1984, ApJ, 285, 89

Dullemond, C. P. 2002, A\&A, 395, 853

Dullemond, C. P., Dominik, C., \& Natta, A. 2001, ApJ, 560, 957

Dullemond, C. P., van den Ancker, M. E., Acke, B., \& van Boekel, R. 2003, ApJ, 594, 47

Dutrey, A. 2004, in Star formation at high angular resolution, IAU Symp., 221

Dutrey, A., Guilloteau, S., \& Simon, M. 1994, A\&A, 286, 149

Eisner, J. A., Lane, B. F., Akeson, R. L., Hillenbrand, L. A., \& Sargent, A. I. 2003, ApJ, 588, 360

Glindemann, A., Algomedo, J., \& Amestica, R. 2003, SPIE, 4838, 89

Grady, C. A., Polomski, E. F., Henning, Th., et al. 2001, AJ, 122, 3396

Henning, Th., Launhardt, R., Steinacker, J., \& Thamm, E. 1994, A\&A, 291,546

Herbig, G. H. 1960, ApJS, 131, 516

Herbst, W., \& Shevchenko, V. S. 1999, AJ, 118, 1043

Hillenbrand, L. A., Strom, S. E., Vrba, F. J., \& Keen, J. 1992, ApJ, 397,613

Keller, L. P., Hony, S., \& Bradley, J. P. 2002, Nature, 417, 148

Kenyon, S. J., \& Hartmann, L. 1987, ApJ, 323, 714

Laor, A., \& Draine, B. T. 1993, ApJ, 402, 441

Leinert, Ch., Richichi, A., \& Haas, M. 1997, A\&A, 318, 472

Leinert, Ch., Haas, M., Ábrahám, P., \& Richichi, A. 2001, A\&A, 375, 927

Leinert, Ch., Graser, U., Graser, U., \& Waters, L. B. F. M. 2003, SPIE, 4838,893

Liu, W. M., Hinz, P. M., Meyer, M. R., et al. 2003, ApJ, 598, L111

Lucy, L. B. 1974, AJ, 79, 745

Malfait, K., Waelkens, C., Waters, L. B. F. M., et al. 1998a, A\&A, 332, L25

Malfait, K., Bogaert, E., \& Waelkens, C. 1998b, A\&A, 331, 211

Malfait, K., Waelkens, C., Bouwman, J., de Koter, A., \& Waters, L. B. F. M. 1999, A\&A, 345, 181

Mannings, V., \& Sargent, A. I. 1997, ApJ, 490, 792

Mattila, K., Lemke, D., Hailala, L. K., et al. 1996, A\&A, 315, L353

Meeus, G., Waters, L. B. F. M., Bouwman, J., et al. 2001, A\&A, 365, 476

Men'shchikov, A. B., Henning, Th., \& Fischer, O. 1999, ApJ, 519, 257

Millan-Gabet, R., Schloerb, F. P., \& Traub, W. A. 2001, ApJ, 546, 358

Natta, A., \& Whitney, B. A. 2000, A\&A, 364, 633

Natta, A., Grinin, V. P., \& Mannings, V. 2000, in Protostars and Planets IV, ed. V. Mannings, A. P. Boss, \& S. S. Russell, 559

Pérez, M. R., van den Ancker, M. E., de Winter, D., \& Bopp, B. W. 2002, A\&A, 416, 647

Richichi, A., \& Percheron, I. 2002, A\&A, 386, 492

Roelfsema, P. R., Cox, P., Tielens, A. G. G. M., et al. 1996, A\&A, 315, L289

van den Ancker, M. E., de Winter, D., \& Djie, H. R. E. T. A. 1999a, A\&A, 330, 145

van den Ancker, M. E., Wesselius, P. R., Tielens, A. G. G. M., van Dishoeck, E. F., \& Spinoglio, L. 1999b, A\&A, 348, 877

van den Ancker, M. E., Meeus, G., Cami, J., Waters, L. B. F. M., \& Waelkens, C. 2001, A\&A, 369, 17

van Boekel, R., Waters, L. B. F. M., Dominik, C., et al. 2003, A\&A, 400, L21

van Boekel, R., et al. 2004, A\&A, in press 